
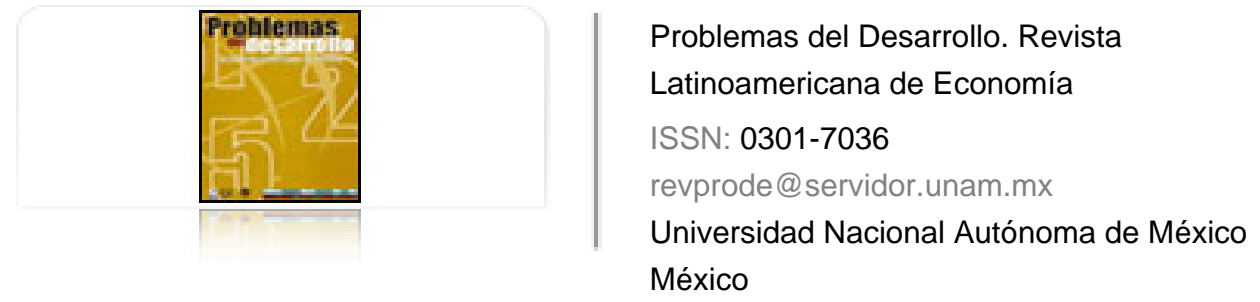

Miguel Velasco, Andrés E.; Heredia González, Armando

Regiones, competitividad y desarrollo en México

Problemas del Desarrollo. Revista Latinoamericana de Economía, vol. 35, núm. 138, 2004, pp. 11-31

Universidad Nacional Autónoma de México

Distrito Federal, México

Disponible en: http://www.redalyc.org/articulo.oa?id=11825948002

- Cómo citar el artículo

- Número completo

- Más información del artículo

Página de la revista en redalyc.org

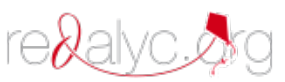

Sistema de Información Científica

Red de Revistas Científicas de América Latina, el Caribe, España y Portugal Proyecto académico sin fines de lucro, desarrollado bajo la iniciativa de acceso abierto 


\section{REgiones, COMPETITIVIDAD Y desarRollo en MÉXICO}

\section{Andrés E. Miguel Velasco* Armando Heredia González ${ }^{* *}$}

Fecha de recepción: 12 de octubre de 2002. Fecha de aceptación: 1 de junio de 2004.

\section{Resumen}

El artículo analiza la relación existente entre la competitividad y el desarrollo regional de México durante los periodos 1990-1995 y 1995-2000. Se apoya en el supuesto de que al inicio del siglo XXI la competitividad regional en este país aún depende de la presencia de factores clásicos de la organización espacial csomo la infraestructura o de políticas públicas como el Plan Puebla-Panamá. Sin embargo, no debe esperarse que la existencia o impulso de estos factores necesariamente conduzca o se refleje en un mayor desarrollo, lo cual implica que la competitividad pueda no ser un factor fundamental para el impulso al desarrollo regional en México.

Palabras clave: región, competitividad, desarrollo regional, Plan Puebla-Panamá, Sur-sureste.

Abstract

The article analyzes the existing relationship between competitiveness and regional development in Mexico during the periods 1990-1995 and 1995-2000. It is supported by the assumption that at the beginning of the 21 st century regional competitiveness in this country still depends on the presence of classical factors of spatial organization including infrastructure or public policies such as the Plan Puebla-Panama. However, it should not be expected that the existence or stimulus of these factors necessarily leads to or reflects greater development, implying that competitiveness may not be a fundamental factor for sparking regional development in Mexico.

Key words: Region, competitiveness, regional development, Plan Puebla-Paname, southsoutheast.

* Profesor-investigador del Instituto Tecnológico de Oaxaca, México. Correo electrónico: andmig56@hotmail.com

* Profesor-investigador del Instituto Tecnológico de Oaxaca, M éxico. 


\section{Résumé}

L'article analyse la relation entre la compétitivité et le développement régional du Mexique pendant les périodes de 1990-1995 et 1995-2000. Il s'appuie sur le supposé de la dépendance, au début du siècle, de la compétitivité régionale dans ce pays de la présence de facteurs classiques d'organisation spatiale tels que l'infrastructure ou les politiques publiques comme le Plan Puebla-Panama. Cependant, il ne faut pas espérer que l'existence ou l'élan de ces facteurs conduise nécessairement ou se reflète par un plus grand développement, ce qui implique que la compétitivité peut ne pas être un facteur fondamental de l'élan donné au développement régional au Mexique.

Mots clés: région, compétitivité, développement régional, Plan Puebla-Panama, Sud-Sud Est.

\section{Resumo}

Uma análise da relação existente entre a competitividade e o desenvolvimento regional do México, durante os periodos 1990-1995 e 1995-2000. Este artigo foi concebido a partir da constatação de que no início do século XXI a competitividade regional no México ainda depende da presença de fatores clássicos da organização espacial, como infra-estrutura ou políticas públicas - vide Plano Puebla-Panamá. Porém, adverte que não se deve esperar que a existência ou impulsão desses fatores necessariamente conduza ou se reflita em um maior desenvolvimento, o que pode sugerir que a competitividade não seja um fator fundamental para o desenvolvimento regional no México.

Palavras-chaves: região, competitividade, desenvolvimento regional, Plano Puebla-Panamá, Sul-sudeste. 


\section{La conceptualización de la relación competitividad-desarrollo regional}

$\mathrm{E}$

l término desarrollo sugiere la capacidad que tienen las sociedades modernas para crecer en todos los sentidos. Desde el punto de vista social, el desarrollo puede

concebirse como el "proceso permanente de mejoría en los niveles de bienestar social, alcanzado a partir de una equitativa distribución del ingreso y la erradicación de la pobreza, observándose índices crecientes de mejoría en la alimentación, educación, salud, vivienda, medio ambiente y procuración de justicia en la población" (SHCP, 2001).

Haciendo énfasis en el aspecto regional, el desarrollo puede entenderse como "el proceso de cambio sostenido, que tiene como finalidad el progreso permanente de la región, de la comunidad regional como un todo y de cada individuo residente en ella" (Boisier 1996). Ahondando en este concepto, puede decirse que se le considera en dos vertientes: a) subordinado al contexto nacional, y $b$ ) independiente de ese contexto. En el primer caso, el desarrollo regional es un proceso de desarrollo nacional a escala regional (subnacional), que abarca las características económicas, sociales y físicas del cambio en una zona durante un determinado periodo (onU, 1972), identificándose los siguientes objetivos en su consecución: $i$ ) un nivel adecuado y creciente de eficiencia basado en la incorporación orgánica de los recursos naturales y humanos, y en un manejo estratégico del espacio económico y social; ii) una distribución territorial adecuada de los esfuerzos y beneficios del desarrollo, en busca de la eliminación de los contrastes regionales adversos, iii) relaciones interregionales, orgánicas y justas, $i v$ ) condiciones reales de un desarrollo interior autosostenido y creciente; $v$ ) una ocupación y un desarrollo metódico para orientar y facilitar el desarrollo de los asentamientos humanos y de la vida en comunidad; y vi) la incorporación sistematizada de la iniciativa y de la participación popular y local. En la segunda vertiente, el desarrollo regional se concibe como: a) un aumento del bienestar en la región expresado en indicadores tales como el ingreso por habitante, su distribución entre la población, la disponibilidad de servicios sociales y la adecuación de sus normas legales y administrativas; $b$ ) procesos de transformación económica y social que comprenden crecimiento del producto per capita, cambio de las estructuras productivas y modernización de la sociedad en su conjunto. Entre otras cosas, se manifiesta a través del equilibrio entre las regiones (Ortega, 1982).

Recientemente, con la visión de que la generación de riqueza no es un fin en sí mismo, en las regiones también se trata de privilegiar el impulso al "desarrollo sustentable" (CMMAD, 1988), definido como el que satisface las necesidades de las generaciones presentes $\sin$ 
comprometer la sobrevivencia de las generaciones futuras; como el desarrollo humano, el cual se concibe como el "proceso conducente a la ampliación de las opciones de las personas en todas las esferas". Desde esta perspectiva, el desarrollo carece de sentido si repercute negativamente en el ambiente y los recursos naturales, y si no se refleja en las capacidades humanas y en su ampliación permanente, lo cual implica que las personas aumenten su productividad, participen en el proceso de generación de ingresos y en el empleo remunerado; tengan acceso equitativo a las oportunidades para que puedan beneficiarse de ellas; protejan las oportunidades de vida de las generaciones futuras, y promuevan el respeto a los ecosistemas (CONAPO, 2001).

Cabe señalar que las expectativas creadas por el concepto desarrollo no han encontrado una comprobación plena en la mayoría de las regiones de los países en vías de desarrollo o de economías emergentes como todavía se considera a México. Por tal motivo, muchas regiones de este país, como el Sur-Sureste, aún transitan en busca de los medios o factores para acelerar su desarrollo. Uno de ellos es el mercado, básicamente por medio de los mecanismos de generación de competencia y, en los últimos años, de competitividad. De aquí deriva la utilidad de analizar cuál es la relación que el desarrollo mantiene con ésta última.

La primera reflexión al respecto conduce a reafirmar que una región es un sistema complejo que delimita el espacio vital de las sociedades y está conformada por una diversidad de factores de tipo económico, social, cultural y ambiental en interacción. En principio, la competitividad de las regiones está condicionada por la convivencia de los elementos heterogéneos de la región, lo cual implica tomar en cuenta que la citada interacción de los mismos no siempre puede resultar armónica, sino en muchas ocasiones caótica, como sucede cuando la actividad humana en lugar de propiciar el desarrollo armonioso esperado, ocasiona el deterioro de los recursos naturales y, por ende, menoscaba el bienestar de la sociedad.

La visión armónica del desarrollo regional descarta o considera en un segundo nivel los desórdenes que puede ocasionar la competencia o los intentos por lograr la competitividad deseada, pues interpreta el comportamiento regional a partir del principio de las "ventajas comparativas y la especialización regional" de David Ricardo. Este autor señaló que la especialización en los tipos de producción — para los cuales las regiones tienen una ventaja comparativa fundada en el mejor aprovechamiento de sus recursos- puede dar como resultado mayores ingresos para todos los que participan en el comercio. Este es el argumento clásico para justificar el libre comercio entre las regiones y las naciones. Tales ventajas serían resultado de la acumulación de los recursos humanos o de capital (manufacturas e infraestructura), tales como mano de obra especializada, edificios industriales, sistemas de transportación, redes de comunicación, sistemas educativos, industrias tecnológicamente desarrolladas, entre otros. La idea subyacente en este principio es que la especialización con los diferentes tipos de producción, para los que los lugares poseen una 
ventaja comparativa, traería un aumento de la riqueza para las regiones involucradas. En caso de lograrse una aceptable competitividad, ésta supone que la misma tendría un impacto favorable o armónico en la región donde se manifieste.

Ahora bien, algunos autores consideran que en el mundo actual "no compiten empresas sino sistemas" (Rosales, 1994).Y aunque se presupone que la empresa es el nudo crucial de la competitividad y la innovación, está integrada a una red de vinculaciones que incluyen a sus proveedores de bienes y servicios, al sector financiero, al sistema educativo, tecnológico, energético, de transportes, de telecomunicaciones (entre otros) así como la infraestructura y la calidad del sector público y de las relaciones al interior de las propias regiones.

Los cambios observados a partir de la década de los ochenta en el contexto internacional están caracterizados por el fenómeno de la globalización de la economía y, por consiguiente, "en este mundo globalizado no se compite bajo el esquema tradicional de empresa versus empresa, sino en uno nuevo de cadena empresarial versus cadena empresarial, cluster versus cluster, región versus región, país versus país" (Villareal y Villareral, 2002). Se genera una serie de impactos regionales y locales teniendo como aspectos sobresalientes la apertura de las economías, la intensificación del uso de la tecnología de la información en los procesos productivos, la mayor calificación de los recursos humanos, así como el desarrollo de los encadenamientos mercantiles. En este sentido, Yoguel (2000) postula "que las ventajas comparativas de los países, regiones y agentes no se derivan necesariamente de su dotación factorial, sino también de factores intangibles que se construyen a partir del desarrollo de competencias endógenas y de la articulación con otros agentes", los cuales son el sustento de la competitividad.

Una de sus primeras definiciones cercanas al aspecto regional es la Scott y Lodge (1985), quienes señalan que la "competitividad de una nación es un asunto de la estrategia económica y que la teoría de las ventajas comparativas ya no se puede considerar adecuada como una base para el diagnóstico y la determinación de políticas" (citado en López, 1999). Porter (1990) define la competitividad como la producción de bienes y servicios de mayor calidad y de menor precio que los competidores domésticos e internacionales, manifestándose en crecientes beneficios para los habitantes de una nación al mantener y aumentar los ingresos reales.

La CEPAL (1992) considera que la competitividad internacional requiere de una transformación productiva basada en la creciente difusión e incorporación del progreso técnico al proceso productivo. El progreso técnico es el factor que posibilita el crecimiento con equidad y da viabilidad a la convergencia de la competitividad con la sustentabilidad ambiental. La incorporación del progreso técnico al proceso productivo requiere fortalecer la infraestructura tecnológica, la base empresarial y la calidad de los recursos humanos; requiere, asimismo, políticas que faciliten el aprendizaje tecnológico y la articulación productiva, y reconozcan el carácter sistémico de la competitividad. Por consiguiente, 
es de suponerse que se ha estado gestando el cambio del paradigma de competitividad pasando de las ventajas comparativas a las competitivas de las industrias y regiones.

Porter (1985) establece la diferencia entre ventajas comparativas y competitivas en países e industrias. La teoría de las comparativas tiene como objeto de análisis a los países y regiones, enfatizando su estudio en aspectos económicos, descuidando elementos específicos de la estructura de producción y organización internas de las empresas y las propias regiones. Las ventajas comparativas promueven en las regiones las actividades con abundancia de recursos para producir con costos y precios menores que sus competidores, sugiriéndoles participar en el mercado. Hoy en día, la dinámica de la actividad de las empresas y las regiones en el mercado y en el contexto internacional está en función de su velocidad de innovación, esto es, del número de nuevos productos introducidos por unidad de tiempo y de la rapidez de imitación de las innovaciones de los países extranjeros (Loyola y Schettino, 1994), así como en el mejoramiento tecnológico, y en su capacidad de acceso a los recursos públicos y privados.

Para Porter $(1990,1998)$ un concepto significativo de la competitividad en el ámbito nacional tiende a ser la productividad. El principal objetivo de una nación es proporcionar un nivel de vida alto y creciente a sus ciudadanos. La habilidad para lograr eso depende de la productividad con la cual se emplea la fuerza de trabajo y el capital de un país. La competitividad depende, hoy en día, cada vez menos de la disminución de costos en función de la abundancia de los recursos productivos, y cada vez en mayor medida en el conocimiento (científico, tecnológico y sistemas de información), y en la gestión de la tecnología (del producto, del proceso productivo, del proceso organizativo). Por tanto, la competitividad se basa cada vez menos en las ventajas comparativas y más en las competitivas, las cuales se generan al interior de las empresas y las regiones que las cobijan (Rosales, 1991).

Loyola y Schettino (1994) se preguntan, ¿por qué algunos países consiguen ser más competitivos que otros? La respuesta se encuentra en un artículo titulado "La ventaja competitiva de las naciones" de Michael Porter (1990) en el cual el autor señala que la "prosperidad de una nación se crea no se hereda". En este sentido, la competitividad de una nación depende de la capacidad de innovación de su industria y, de acuerdo con el pensamiento predominante, los costos laborales, las tasas de interés, las tasas de intercambio y las economías de escala son los más importantes determinantes de la competitividad.

Otro enfoque para comprender la competitividad de las regiones es el hecho de que las organizaciones o países frecuentemente se identifican con el incremento de la parte del mercado de exportación del que llegan a posesionarse o un alto retorno de las inversiones que proporcionan sus economías. Por tanto, el éxito en la competitividad de las regiones para participar en los mercados globales depende de la innovación a través de la producción de nuevos productos y servicios, sin embargo, su sostenibilidad a largo plazo 
también depende de los aspectos organizacionales, de la cooperación entre instituciones (proveedores, consumidores, universidades, bancos, instituciones de transferencia y otros).

La Organización de Cooperación para el Desarrollo Económico (OECD, 1992) define la competitividad como "el grado en el cual un país, bajo condiciones de mercados libres y justas, puede producir bienes y servicios que superen el test de los mercados internacionales, incrementando en forma sostenida los ingresos reales de su población”. La competitividad estructural analizada por la OCDE (1992) se refiere a la especialización de la economía, la innovación tecnológica, la calidad de las redes de distribución y los factores de localización (host) todo lo cual constituye el estado de suministro de bienes y servicios (Hatzichronoglou, 1996). La competitividad así entendida tiende a mejorar el desarrollo de las economías y, en particular, el desarrollo de una región (López, 1999).

Por otra parte, el International Institute for Management Development (IIMD, 1997) define la competitividad como "la capacidad que tiene un país o una empresa para, proporcionalmente, generar más riqueza que sus competidores en mercados internacionales". La Comisión Económica para América Latina y El Caribe (CEPAL) considera que "la competitividad auténtica debe estar basada en la incorporación de la tecnología y el uso renovable de los recursos naturales, concepción que contrasta con la competitividad espuria que se basa en la explotación de los recursos humanos y naturales".

Con base en las definiciones anteriores se deducen dos aspectos: a) que la competitividad se asocia a diferentes áreas geográficas, sean empresas, industrias o, en un nivel más amplio, países o regiones; $b$ ) la competitividad está muy asociada al concepto de productividad, en el sentido de que un mayor rendimiento de los recursos naturales, de la mano de obra y del capital son un camino indispensable, aunque no necesariamente suficiente para lograr que un país o región logre aumentar su competitividad (Ministerio del Interior, 2000). Si se toma en cuenta que las acciones emprendidas por las empresas exitosas no necesariamente se difunden en el comportamiento de las otras empresas, se requiere un concepto más dinámico, el de la competitividad sistémica, en el cual se pueden observar actividades intra e interempresariales y con instituciones de apoyo como indicadores de la competitividad. En este último aspecto adquiere importancia la región. Desde esta perspectiva, la competitividad puede definirse como "la capacidad de las regiones para alcanzar los niveles de crecimiento sustentables en el tiempo" (Ministerio del Interior, 2000).

La competitividad regional puede entenderse también como la capacidad de una región para adelantarse a otras en el acceso al conocimiento y a la tecnología, a los recursos públicos y privados (materiales y financieros), así como al mercado para la venta de sus productos o la adquisición de recursos naturales y materias primas. La región que en un momento consigue adelantar a las demás, genera un aumento de su desarrollo, mejora su acceso a los recursos públicos y privados, y asimismo logra una presencia un poco más segura en el mercado, lo cual le permite hacerse, durante cierto tiempo, de mayores recursos privados 
y públicos, reinvirtiéndolos con la perspectiva de obtener nuevas ventajas, que necesitará para mantenerse o avanzar en su posición en el contexto regional (Lengnick, 1992).

De alguna manera, inicialmente estas ventajas tienen que sustentarse con la presencia de los factores clásicos del desarrollo regional, pero debe quedar claro que la competitividad no es algo inherente a las regiones, sino resultado de políticas públicas y privadas que buscan el impulso del mercado y de las empresas que las regiones albergan, pues el propósito de la región no es la competencia o la competitividad como tal, sino asegurar el espacio vital de los seres vivos que la habitan. De ahí la importancia de analizar cuál es el papel de estos factores en la competitividad y el desarrollo regional.

\section{Los factores clásicos del desarrollo regional y la competitividad}

La teoría clásica del desarrollo regional propone diversos factores, destacando inicialmente los de tipo económico, como la demanda de espacio, el transporte y sus costos, así como las economías de escala para explicar o describir la organización económica de las regiones (Hermansen, 1979).

La interpretación es que la interacción de estos factores - y otros adicionales como los geográfico-ambientales e incluso las políticas públicas- de alguna manera se manifiesta en las ventajas que pueden adquirir las regiones con respecto a las demás, pues la presencia de los factores de la organización espacial influye de manera tal que se tendrá mayor competitividad $(C)$ si se posee: $i$ ) mayor infraestructura, transporte y servicios (I); ii) mejores condiciones económicas $(E)$; iii) mejores condiciones geográficas (ubicación, calidad y usos del suelo) $(G) ; i v$ ) mejores apoyos financieros (privados y públicos) $(F)$; $v$ ) mejores condiciones ambientales (recursos y calidad del ambiente) $(A)$; y vi) mejores condiciones demográficas (como por ejemplo más población capacitada) $(P)$.

La visión clásica da por hecho que una mayor competitividad $(C)$, tarde o temprano, se proyecta en un mayor desarrollo regional $(D)$ o viceversa, pues ambos tienden a ser un reflejo del nivel alcanzado por los factores de competitividad que la región posea, es decir, su contrastación conduce a la propuesta:

$$
\mathrm{D}=\left[\mathrm{f}_{1}(\mathrm{C})=\mathrm{f}_{\mathrm{i}}(\mathrm{I}, \mathrm{E}, \mathrm{G}, \mathrm{F}, \mathrm{A}, \mathrm{P})\right]>0
$$

Esta relación plantea un proceso armónico capaz de generar círculos virtuosos entre el desarrollo y los factores de competitividad que la región posea.

Sin embargo, puede darse el caso de que la competitividad no impacte favorablemente el desarrollo regional, e incluso, sea un activador de círculos viciosos. Ello podría ocurrir si se manifestara la propuesta:

$$
D=\left[f_{2}(C)=f_{h}(I, E, G, F, A, P)\right][0]
$$

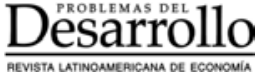


Esta relación plantea que la competitividad $(C)$ no necesariamente deriva de una relación favorable con respecto de todos o alguno de los factores de competitividad (I, $E, T$, $G, F, A, P)$ que pudieran incidir en el desarrollo regional.

Lo anterior podría ocurrir cuando se emprendan acciones orientadas a la actividad económica pero que no toman en cuenta la diversidad de recursos que tienen las regiones (por ejemplo su biodiversidad o multiculturalidad) y que no presentan propuestas para su cuidado, preservación o incluso formas de participación social en los programas regionales.

También pueden suscitarse estos círculos viciosos cuando no se toma en cuenta la problemática social, pues el mundo de las regiones entraña un gran número de conflictos. Éstos pueden ser solamente en el nivel de declaraciones o en operaciones económicas cotidianas (como por ejemplo los problemas operativos o intermedios por las concesiones que implican los programas de desarrollo), hasta conflictos declarados (cuando las regiones o comunidades entran en franca confrontación en la lucha por la tierra o por el manejo de sus recursos naturales y de los mercados, pues ven afectados sus intereses).

La relación armoniosa entre la competitividad y el desarrollo también supone que puedan afectarse o perderse ciertos recursos regionales (flora, fauna, cultura u otras creaciones humanas), considerando esta pérdida como algo temporal. Sin embargo, el impacto de algunos programas para elevar la competitividad podría inducir la alteración del equilibrio ecológico, la emigración (entre otros) en la región, dificultando su desarrollo sustentable.

Es este el contexto conceptual que se toma como referencia para evaluar el comportamiento de la competitividad y el desarrollo del caso concreto de las regiones de México.

\section{Bases metodológicas para el análisis de la competitividad y el desarrollo en México}

Las regiones por analizar en el presente artículo se señalan en el Cuadro 1.

Cuadro 1

Regiones y estados de México

\begin{tabular}{ll}
\hline \multicolumn{1}{c}{ Región } & \multicolumn{1}{c}{ Estados } \\
\hline $\begin{array}{l}\text { Noroeste } \\
\text { Norte }\end{array}$ & Baja California, Baja California Sur, Sinaloa, Sonora. \\
Noreste & Coahuila, Chihuahua, Durango \\
Centro-Norte & Auevo León, Tamaulipas \\
Centro-Occidente & Colima, Guanajuato, Jalisco, Michoacán, Nayarit \\
Centro-Este & Distrito Federal, México, Hidalgo, Morelos, Puebla, Querétaro, Tlaxcala \\
Sur & Chiapas, Guerrero, Oaxaca \\
Este & Tabasco, Veracruz \\
Península de Yucatán & Campeche, Quintana Roo, Yucatán \\
Sur-Sureste & Integrada por las regiones Sur, Este y Península de Yucatán, incluyendo Puebla \\
\hline
\end{tabular}

Fuente: Ángel Bassols Batalla, "Investigaciones urbanas y regionales de México: ¿para conocer o transformar una realidad?", en Humberto Muñoz (coordinador), La sociedad mexicana frente al tercer milenio, Miguel Ángel Porrúa-Coordinación de Humanidades, México, 1999. 
En la comparación entre la competitividad y el desarrollo de las regiones se utilizan los índices de desarrollo $(D)$, y de competitividad $(C)$ durante los periodos 1990-1995 y 1995-2000, obtenido el primero del índice de desarrollo humano (IDH); derivándose el segundo de indicadores de factores como el Geográfico $(G)$, de Infraestructura $(I)$, Económico $(E)$, Financiero $(F)$, Ambiental $(A)$, y Demográfico $(P)$. Estos índices se comparan para valorar el grado de asociación (correlación) existente entre ellos.

La propuesta metodológica concreta es que las proposiciones de las ecuaciones (1) y (2) del apartado anterior puedan corroborarse a través de las condiciones:

$$
\begin{gathered}
\Sigma\left(\operatorname{Corr}\left(\mathrm{D}, \mathrm{V}_{\mathrm{i}}\right)\right)>0 ;(\mathrm{r}+) \\
\Sigma\left(\operatorname{Corr}\left(\mathrm{D}, \mathrm{V}_{\mathrm{i}}\right)\right)=0 ;(\mathrm{r} \text { nula }) \\
\Sigma\left(\operatorname{Corr}\left(\mathrm{D}, \mathrm{V}_{\mathrm{i}}\right)\right)<0 ;(\mathrm{r}-)
\end{gathered}
$$

donde:

$D$ : Desarrollo;

$V_{i}$ : corresponde a los factores de competitividad $C, I, E, G, F, A, P$ comentados en el apartado anterior; $y$

$r$ : es el coeficiente de correlación. Los rangos para valorar las correlaciones positivas se toman de la escala: $0.0 \leq \mathrm{r} \leq 0.2$ muy débil; $0.2 \leq \mathrm{r} \leq 0.4$ débil; $0.4 \leq \mathrm{r} \leq 0.6$ moderada; $0.6 \leq \mathrm{r} \leq 0.8$ fuerte; y $0 . \leq \mathrm{r} \leq 1.00$ muy fuerte.

La propuesta es que existe una relación armónica o un impacto favorable de la competitividad en el desarrollo si la relación manifiesta la condición (3), así como asociaciones fuertes y muy fuertes. No existe una relación favorable entre la competitividad, los factores clásicos y el desarrollo si suceden las condiciones 3.a o 3.b.

Específicamente, el cálculo de los índices empleados se basó efectuando los pasos que se indican:

1) Para el índice de competitividad se obtuvieron los valores absolutos de diversos indicadores durante los periodos 1990-1995 y 1995-2000, agrupándolos por entidad federativa en seis grandes subsistemas (entre paréntesis se señalan los indicadores respectivos de cada uno de ellos): Subsistema Infraestructura (I) [longitud de carreteras (km), y el total de unidades de aeropuertos, puertos, ferrocarriles]; Subsistema Geográfico $(G)$ [distancia $(\mathrm{km})$ al D.F. y hacia la frontera norte]; Subsistema Económico (E) [total de unidades de manufacturas, total comercios, servicios financieros, administrativos y bienes inmuebles, servicios comunales y sociales]; Subsistema Financiero $(F)$ [\$ por habitante de participaciones federales y el producto interno bruto per capita]; Subsistema Ambiental (A) [total de especies de flora y fauna]; y el Subsistema Demográfico $(G)$ [número de habitantes población total, población económicamente activa ocupada, población ocupada industrial y población ocupada como profesionistas y técnicos].

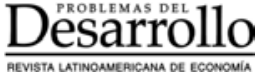


Con los valores de los indicadores se calcularon los índices de los subsistemas, utilizando como datos la proporción de cada estado con respecto al valor nacional, agrupándose posteriormente los resultados de los índices por estado y se obtuvieron los promedios correspondientes para las regiones de México indicadas en el Cuadro 1.

La descripción aritmética de los índices es:

$$
I_{j}=S F p_{i}=S\left(X_{i j}-X_{i}\right) / d_{i} j=1 \ldots n
$$

en donde:

$I_{j}:$ índice del conjunto de indicadores;

$X_{i j}$ : valor del indicador i;

$X_{j}:$ valor de la media del indicador $i$

$d_{j}$ : desviación estándar del indicador $i$;

$F p_{i}$ : factor de ponderación del indicador $i$, la cual indica que los índices se obtienen de la combinación de los indicadores de los diversos factores $\left(I_{i}\right)$ a través del método de componentes principales.

2) Se define la:

Ganancia de competitividad $=$ Suma de los factores positivos de competitividad Pérdida de competitividad $=$ Suma de los de factores negativos de competitividad

entendiéndose por factor positivo de competitividad aquél que logró tasas de crecimiento positivas, o como factor negativo de competitividad el que alcanzó tasas de crecimiento negativas, durante los periodos analizados.

Para obtener la ganancia o pérdida de competitividad:

a) Se determina el crecimiento de los índices de competitividad de los factores durante el periodo 1990-1995 y 1995-2000; b) con base en las tasas anteriores se contabiliza la frecuencia de los factores que lograron tasas de crecimiento positivas o negativas; $c$ ) para determinar el impacto de los factores en el cambio de la competitividad, se considera la siguiente escala:

Escala de comparación de ganancia de competitividad (Factores con cambio positivo)

Ganancia rápida: 3 factores

Ganancia media: 2 factores

Ganancia lenta: 1 factor

Sin ganancia: 0 factores
Escala de comparación de pérdida de competitividad (Factores con cambio negativo)

Pérdida muy rápida: 5 factores

Pérdida rápida: 4 factores

Pérdida media: 3 factores

Pérdida lenta: 2 factores 
3) El índice de desarrollo $(D)$ fue tomado directamente como un dato proporcionado por el Consejo Nacional de Población, considerando como indicador el índice de desarrollo humano (IDH), el cual es una medida innovadora y útil, que al ser un indicador compuesto, es comparable internacionalmente, combinando la longevidad (medida mediante la esperanza de vida al nacer), el logro educacional (a través de la alfabetización de adultos y la matrícula combinada de varios niveles educativos), y el nivel de vida, mediante el producto interno bruto anual per capita ajustado (paridad del poder adquisitivo en dólares). El IDH es una medida de potenciación, la cual indica que los individuos, cuando disponen de esas tres oportunidades básicas, están en condiciones de tener acceso a, y aprovechar otras opciones (CONAPO, 2001).

4) Posteriormente se efectuó la correlación entre los índices considerados como variable independiente (incluido el índice de competitividad), con el de desarrollo (variable dependiente), es decir:

$$
\left(\operatorname{Corr}\left(\mathrm{D}, \mathrm{V}_{\mathrm{i}}\right)\right)=\mathrm{r}
$$

donde:

$D: \quad$ índice de desarrollo;

$V_{i}$ : índices de las variables independientes que corresponden a los índices (I, $E, T, G$, $F, A, P)$ comentados en el inciso 1 del presente apartado;

Corr : correlación; y

$r \quad$ : coeficiente de correlación.

5) Finalmente se efectuó la ponderación $\left(p_{i}\right)$ del valor de las correlaciones de los índices citados con base en los criterios siguientes:

$$
\begin{aligned}
& \operatorname{Si}\left(\operatorname{Corr}\left(D, V_{i}\right)\right)>0, p_{i}=0 \\
& \operatorname{Si}\left(\operatorname{Corr}\left(D, V_{i}\right)\right)=0, p_{i}=0 \\
& \operatorname{Si~}\left(\operatorname{Corr}\left(D, V_{i}\right)\right)<0, p_{i}=-1
\end{aligned}
$$

La sumatoria de las ponderaciones $\left(\mathrm{p}_{\mathrm{i}}\right)$ de las correlaciones se obtuvo con base en el criterio siguiente. $\mathrm{Si}$

$$
\Sigma \mathrm{pi}=0 \quad(5 \mathrm{~d})
$$

esta proposición indica que la relación entre la competitividad (o sus factores) y el desarrollo regional es armónica; es decir, si mejora cualesquiera de estos factores, el desarrollo regional también mejorará. Si 


\section{Si $\Sigma$ pi $<0 \quad(5 e)$,}

en ésta se indica que la relación entre la competitividad (o sus factores), no necesariamente se refleja en una mejora del desarrollo regional.

El procedimiento metodológico descrito sirve de referencia para la obtención de los resultados numéricos subsecuentes.

\section{La competitividad y el desarrollo del contexto regional en México}

Desde una perspectiva global, México es el país más abierto del mundo, pues mantiene acuerdos de libre comercio con 31 países en tres continentes y presenta un índice de apertura al exterior ${ }^{1}$ de $70 \%$, pero paradójicamente es uno de los países menos competitivos, al ocupar el número 43 dentro de los 59 países en el reporte global de competitividad 2000. Esto indica que los pilares que soportan el crecimiento a mediano plazo han perdido solidez y sustentabilidad con respecto a los otros países (Villareal y Villarreal, 2002).

De igual manera, en el Informe sobre Desarrollo Humano 2000, el Programa de las Naciones Unidas para el Desarrollo ubicó a México en la categoría de "desarrollo humano medio". El país ocupaba el quincuagésimo quinto lugar en la clasificación mundial (del total de 174 naciones) y el noveno entre las naciones de desarrollo humano medio. Por otra parte, los promedios nacionales indicaban que en 1997 el Distrito Federal, Nuevo León, Baja California Sur, Baja California y Sonora ocupaban las primeras cinco posiciones de acuerdo con el valor registrado por el índice de desarrollo humano. A su vez, estados como Puebla, Guerrero, Oaxaca y Chiapas de la región Sur-Sureste se ubicaban en los últimos cinco lugares de la clasificación nacional (CONAPO, 2001). En lo que respecta a las regiones, las de mayor desarrollo relativo eran la región Noreste (con un índice de desarrollo humano de 0.84), Noroeste (0.82), Península (0.81) y Centro-Este (0.80); seguidas de las regiones Centro-Norte (0.79), Centro-Occidente (0.78), Sur (0.77), Norte (0.77) y Este (0.76). El Sur-Sureste poseía un valor promedio de 0.79.

Por lo que respecta a la competitividad de las regiones mexicanas, se observó que durante los periodos 1990-1995 y 1995-2000, Norte, Noreste y Sur mantuvieron una alta competitividad, mientras que las Noroeste, Centro-Norte, y la Península de Yucatán manifestaron una baja. Otras, como la Centro-Occidente pasaron de media a baja; la CentroEste de alta a media; y la región Este de una competitividad media a baja (Cuadro 3).

Otros cambios ocurridos en la competitividad de las regiones mexicanas durante los periodos analizados pueden resumirse en las siguientes observaciones (cuadros 3 y 4):

1 Índicede apertura $=[($ exportaciones + importaciones $) / \mathrm{PIB}]$. 
a) Las regiones con una competitividad media manifestaron el comportamiento más inestable (cambiante) durante los periodos analizados;

b) Todas las regiones mantuvieron su competitividad ambiental sin cambio; todas disminuyeron su competitividad en lo que respecta a los factores geográficos, y también todas las regiones aumentaron su desarrollo;

c) Las regiones que aumentaron su competitividad por cambios en sus factores demográficos fueron la Noroeste, Norte, Este y la Península de Yucatán;

d) Las regiones que aumentaron su competitividad gracias a su actividad económica fueron la Noroeste, Norte, y Centro-Norte;

e) Las regiones que aumentaron su competitividad en infraestructura fueron la Norte, Centro-Occidente, Sur, Este, y Sur-Sureste;

f) Las regiones que aumentaron en competitividad por apoyos públicos fueron la Noroeste y Centro-Occidente; y finalmente,

g) Las regiones que aumentaron su competitividad global fueron la Noroeste, CentroOccidente, Centro-Este, Este y Sur-Sureste.

El balance en términos de ganancia o pérdida de competitividad durante el periodo 1995-2000 puede describirse señalando que (Cuadro 4):

1) Las regiones sin ganancia o con pérdida muy rápida de competitividad fueron la Noreste y Centro-Este;

2) Las regiones de ganancia lenta o con pérdida rápida de competitividad fueron la CentroNorte, Sur, Península, y la región Sur-Sureste;

3) Las regiones de ganancia media o con pérdida media de competitividad fueron la Centro-Occidente y la región Este; y

4) Las regiones de ganancia rápida o con pérdida lenta de competitividad fueron las regiones Norte y Noroeste.

De igual manera, la valoración de la relación existente entre la competitividad y el desarrollo de las regiones de México durante los periodos analizados puede resumirse en las siguientes observaciones:

i) Durante el periodo 1990-1995, los factores de competitividad más influyentes en el impulso al desarrollo regional fueron el demográfico y los apoyos públicos, aunque con una débil (0.26) y muy débil (0.18) correlación. En el periodo 1995-2000, estos factores fueron los apoyos públicos y el económico, entonces con una fuerte $(0.67)$ y una moderada (0.48) correlación respectivamente (Cuadro 2).

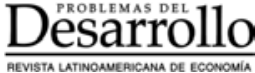


ii) Algunos factores de competitividad manifestaron una relación con el desarrollo contraria a la propuesta armoniosa de la teoría clásica. Así, durante el periodo 1995-2000 el índice geográfico indicaba que la distancia geográfica de las regiones hacia el centro o la frontera del país se manifestaba en menos desarrollo (con una correlación muy débil, $r=-0.12$ ). Lo mismo indicaba el índice de infraestructura, el cual, aunque posee una débil correlación (-0.28), sugería que más infraestructura no necesariamente se reflejaba en un mayor desarrollo regional. El índice ambiental reveló que a mejores condiciones ambientales menos desarrollo (con una correlación moderada, $r=-0.58$ ); y finalmente el índice demográfico, sugiere que existe una relación inversa entre la cantidad de población capacitada y el desarrollo (con una débil correlación, $r=-0.39$ ).

iii) En general, la competitividad y el desarrollo mantuvieron una muy débil (0.10) y débil (0.23) correlación en los periodos 1990-1995 y 1995-2000 respectivamente, lo cual indica que en México el desarrollo de sus regiones ha estado acompañado cada vez de una mayor competitividad, pero con un impacto poco significativo de ésta en el desarrollo regional.

\section{Conclusiones: las perspectivas de desarrollo de la región Sur-Sureste en el contexto del Plan Puebla-Panamá}

Tomando en cuenta las correlaciones (Cuadro 2) de los diversos factores que dan origen a la competitividad regional, así como el valor resultante de la sumatoria de sus ponderaciones que en los periodos 1990-1995 y 1995-2000 resultó negativa (-4), la conclusión general es que en México se cumple el supuesto de que la competitividad no necesariamente es un factor que impulsa significativamente el desarrollo de sus regiones.

Algunas de las razones probables pueden ser, por ejemplo, que la competitividad en las regiones de México todavía hace referencia a: a) los nichos de competitividad de las grandes empresas ubicadas regionalmente, pero que no transfieren sus beneficios al resto de la región; $b$ ) que los programas e instituciones oficiales regionales no guardan una estrecha relación con los programas e instituciones que tienen a su cargo la competitividad; c) que de existir en las regiones, no se ha logrado crear una competitividad sistémica, sino aislada; $y d$ ) que si bien la competitividad puede impactar en algo el aumento del desarrollo, es éste el que en realidad tiende a respaldar una mayor competitividad, por lo cual debería impulsársele con una mayor decisión en las regiones de México.

En este contexto puede destacarse el caso de la región Sur-Sureste, la cual se mantuvo estable durante los periodos analizados con una competitividad media y un nivel de desarrollo bajo. También se observó que la competitividad de esta región destacaba en los factores geográfico y ambiental (el nivel de su índice es alto en estos casos) (Cuadro 3). Sin embargo, durante el periodo 1995-2000 la correlación del primer factor es muy débil y operó en sentido opuesto al desarrollo; es decir, que las mejores condiciones geográficas 
manifestaban un impacto negativo poco significativo en el desarrollo. Por su parte, durante los mismos periodos el factor ambiental poseía una correlación moderada, pero en el sentido contrario al propuesto por la teoría; en otras palabras, que en lugar de contribuir, el desarrollo jugaba un papel opuesto al cuidado o preservación de los recursos ambientales y naturales. Durante el periodo analizado la región Sur-Sureste manifestó una pérdida rápida de su competitividad respecto de otras regiones de México. Por consiguiente, puede considerarse que los factores de la competitividad tampoco han sido relevantes para propiciar el desarrollo de esta región.

Por lo tanto, ¿cuáles pueden ser las perspectivas de esta región en México al inicio del siglo XXI? Las expectativas actuales más importantes de desarrollo para esta región son las perfiladas en las políticas públicas derivadas del Plan Puebla-Panamá (PPP), el cual rebasa las fronteras mexicanas, e implica la participación de los países centroamericanos. Como se ha dado a conocer, éste tiene como objetivo "mejorar la calidad de vida de los habi-tantes de la región". El ppp pretende impulsar su estrategia de desarrollo "a través de dos vertientes: 1) acciones estratégicas resultantes del proceso de planeación regional, integral y sustentable, y 2) programas complementarios concurrentes en la región" (OPEDER, 2001).

El escenario que sustenta el PPP es armonioso, y plantea una situación favorable en todos los aspectos considerados, esto es, en el sentido en el cual se supone que dicho Plan favorecerá una competitividad regional que no impactará negativamente la ecología, la multiculturalidad, y que impulsará las posibilidades de desarrollo de la región.

Sin embargo, una primera reflexión es que si el contexto en el cual se presupone se elevará la competitividad no es precisamente armonioso, el impacto de la competitividad puede conllevar aspectos desfavorables, activando conflictos locales, microregionales, inter e intraregionales que pueden acrecentar la emigración hacia el exterior, la pobreza y las desigualdades sociales existentes en el Sur-Sureste.

De ocurrir lo anterior, el impacto de la competitividad podría ocasionar cambios ambientales y económicos en la región, debido a la problemática que puede acarrear la competencia por los mercados y recursos naturales. El riesgo de la destrucción de su diversidad, así como de la activación de viejos y la generación de nuevos conflictos es alto en esta región de México. Desde esta perspectiva son posibles las propuestas de un escenario que plantea una situación difícil en el sentido de que dicho Plan no impactará favorablemente todo el sur-sureste mexicano, sino que preferentemente posesionaría en los mercados de la región a las grandes empresas y a los interesados en los recursos naturales (agua, petróleo, flora y fauna) que aún posee el Sur-Sureste. A pesar de sus propuestas poco optimistas, este escenario plantea el desafío de poner atención en un manejo cuidadoso de la multiculturalidad, la biodiversidad, así como de la soberanía que posee esta región.

Otra variante adicional podría ser que a partir de la aplicación del PPP se desarticula la región Sur-Sureste en varias microregiones. Éstas — sin lograr necesariamente más altos 
niveles de desarrollo - forman jerarquías, diferencias y desigualdades regionales internas notorias a partir de la aplicación del Plan, pues pueden entrar en franca competencia entre sí, primero por la influencia y recursos que pueda aportar el Plan, y posteriormente en los sectores propios de la región Sur-Sureste, como la lucha por elevar la competitividad entre los centros turísticos, culturales, por el mercado de los recursos naturales producidos en las diversas microregiones (entre otros), creando desigualdades e inequidades a favor de las ciudades, empresas y grupos sociales más influyentes, incluso ajenos a la región.

Esto exige generar opciones que permitan prever que si se acepta la posibilidad que el PPP no impactará favorablemente todas las posibilidades de desarrollo de los estados y microregiones del Sur-Sureste. Debe plantearse una situación en la que puedan aprovecharse las oportunidades que deriven del Plan, pero a partir de las expectativas propias de la población regional, lo cual puede obligar a las instituciones, ciudades, empresas, universidades y gobiernos, a formar redes para aprovechar las oportunidades; así como protegerse de los posibles efectos negativos del proceso de desarrollo logrado hasta ahora. Es decir, esto requiere de "la redefinición de un proyecto político incluyente que promueva una amplia participación social en las decisiones a fin de llevar a cabo las estrategias necesarias en aras de una organización socioterritorial más justa" (Delgadillo, 2001:42), donde la competitividad realmente resulte una fuerza positiva para impulsar el desarrollo regional.

\section{Bibliografía}

Boisier, Sergio, Modernidad y territorio, Santiago de Chile, Instituto Latinoamericano y del Caribe de Planeación Económica y Social, CEPAL, 1996.

CEPAL (Comisión Económica para América Latina), Equidad y transformación productiva: Un enfoque integrado, Santiago de Chile, Naciones Unidas, CEPAL, 1992.

CMMAD (Comisión Mundial del Medio Ambiente y del Desarrollo), Nuestro Futuro Común, Madrid, Alianza Editorial, 1988.

CONAPO, "Desarrollo Humano en México", México, www.conapo.gob.mx, 12 diciembre de 2001.

Delgadillo Macías, Javier; Felipe Torres T. y José Gasca Zamora, "Distorsiones del desarrollo regional de México en la Perspectiva de la Globalización”, Revista Momento Económico, núm. 115, México, IIEC-UNAM, mayo-junio 2001.

Hatzichronoglou, T., Globalisation and competitiveness: Relevant indicators, París, 1996.

Hermansen, Tormod, "Organización espacial y desarrollo económico. Alcances y limitaciones de la planificación espacial”, Doc. D/7, Curso de Planificación Regional del Desarrollo, julio, ILPES-CEPAL, 1979.

IIMD (International Institute for Management Development), World Competitiveness Yearbook, Lausane, IMD, 1997.

Lengnick-Hall, Cynthia A., "Innovation and competitive advantage: What we know and what we need to learn", Journal of Management, vol. 18, núm. 2, 1992.

López Ortega, Eugenio, El concepto de competitividad en el posicionamiento tecnológico, Centro de Investigaciones Interdisciplinarias en Ciencias y Humanidades, México, UNAM, 1999.

Loyola, J. Antonio; Schetttino, Macario Y., Estrategia empresarial en una economía global, México, ITESM-Iberoamericana, 1994.

Ministerio del Interior, "Informe de competitividad regional", Santiago de Chile, Ministerio del Interior, Subsecretaría de Desarrollo Regional y Administrativo, 2000.

OECD, Technology and the economic: the key relationships, París, 1992. 
ONU, The United Nations Programme in Regional Development. A Introduction to Subnational Planning, Nueva York, 1972.

OPEDER (Oficina de Planeación Estratégica y Desarrollo Regional), "Plan Puebla-Panamá (Capítulo México)", México, Gobierno de la República, 17 de Abril de 2001.

Ortega, Arturo, Diccionario de Planeación y Planificación. Un Ensayo Conceptual, Edit. Edicol, México, 1982.

Porter, Michael, Estrategia Competitiva. Técnicas para el análisis de los sectores industriales y de la competencia, México, CECSA, 1985.

,"The competitive advantage of nations", en Michael Porter, On competition, Ed. Harrod Business Review Book, 1998.
"The Adam Smith address: Location, clusters and the "new microeconomics of competition", Business Economics, vol. 33. núm. 1, 1998.

Rosales, Osvaldo, "Competitividad y cambio tecnológico", Revista Interamericana de Planificación (SIAP), vol. XXIV, núm. 96, Santiago de Chile, octubre-diciembre de 1991.

, "Política industrial y fomento de la competitividad", Revista de la CEPAL, núm. 53, Santiago de Chile, 1994.

SHCP (Secretaría de Hacienda y Crédito Público), www.shcp.gob.mx, México, 15 de agosto de 2001.

Villareal, René y Rocío Villareal, México Competitivo 2002, México, Ed. Plaza y Valdés, 2002.

Yoguel, Gabriel, "Creación de competencias en ambientes locales y redes productivas", Revista de la CEPAL, núm. 71, Santiago de Chile, 2000. 


\section{Anexo Estadístico}

México: correlaciones entre los índices de desarrollo y competitividad

\begin{tabular}{lccrr}
\hline \multirow{2}{*}{ Índices } & \multicolumn{2}{c}{ Correlación } & \multicolumn{2}{c}{ Correlación } \\
& $1990-1995$ & Ponderación & $1995-2000$ & Ponderación \\
\hline \multirow{2}{*}{ Geográfico } & -0.08 & -1 & -0.12 & -1 \\
Infraestructura & -0.35 & -1 & -0.28 & -1 \\
Económico & -0.41 & -1 & 0.48 & 1 \\
Apoyos Públicos & 0.18 & 0 & 0.67 & 0 \\
Ambiental & -0.54 & -1 & -0.58 & -1 \\
Demográfico & 0.26 & 0 & -0.39 & -1 \\
Competitividad & 0.10 & 0 & 0.23 & 0 \\
\hline Suma de las ponderaciones & - & -4 & - & -4 \\
\hline
\end{tabular}

Fuente: Los índices fueron elaborados con datos de desarrollo humano proporcionados por CONAPO (2001), Desarrollo Humano en México (www.conapo.gob.mx), 12 de diciembre. La información del PIB fue consultada en INEGI (2002), Producto Interno Bruto por Entidad Federativa, (www.inegi.gob.mx), 14 de febrero. Los datos de población fueron proporcionados por INEGI, Estados Unidos Mexicanos XI y XII Censo General de Población y Vivienda, 1990 y 2000. Algunos informes de la flora y fauna fueron tomados de www.conabio.gob.mx/biocompetitividad/col.htm\#biodiv; 14 de febrero de 2000 (la flora incluye: plantas vasculares, hongos. La fauna incluye únicamente vertebrados mamíferos terrestres, voladores y mamíferos marinos), así como también del Consejo Técnico Consultivo de la Secretaría de Desarrollo Rural y Ecología del Gobierno del Estado de Chiapas (1992), Biocompetitividad. Entre la evolución y la extinción, Gobierno del Estado de Chiapas. Los datos de población ocupada fueron proporcionados por INEGI, (2001) Anuario Estadístico por Entidad Federativa, edición 2001. Los datos de infraestructura fueron consultados en INEGI (1999), Anuario de Estadísticas por Entidad Federativa, edición 1999, Aguascalientes, Mexico; y Subsecretaría de Infraestructura (2002), www.sct.gob.mx, 6 de febrero de 2002. Los documentos de participaciones federales fueron proporcionados por INEGi, Finanzas Públicas Estatales y Municipales de México, 1995-1998, Aguascalientes, México; y El Sol de Acapulco (periódico) (2001), Entrevista Lic. David Colmenares Páramo, presidente del Colegio Nacional de Economistas, Martes 13 de noviembre de 2001, Acapulco, Guerrero, México.

Nota: En la interpretación del cuadro se propone la escala de Salkind como límites para comparar la correlación entre las variables: $0.0 \leq \mathrm{r} \leq 0.2$ muy débil; $0.2 \leq \mathrm{r} \leq 0.4$ débil; $0.4 \leq \mathrm{r} \leq 0.6$ moderada; 0.6 $\leq \mathrm{r} \leq 0.8$ fuerte; y $0 . \leq \mathrm{r} \leq 1.00$ muy fuerte. 
Cuadro 3

Regiones de México: índices de factores, competitividad y desarrollo 1990-1995 / 1995-2000

\begin{tabular}{lcccc}
\hline \multicolumn{1}{c}{ Región } & $\begin{array}{c}\text { Índice } \\
\text { demográfico }\end{array}$ & $\begin{array}{c}\text { Índice } \\
\text { geográfico }\end{array}$ & $\begin{array}{c}\text { Índice } \\
\text { económico }\end{array}$ & $\begin{array}{c}\text { Índice } \\
\text { infraestructura }\end{array}$ \\
\hline Noroeste & Bajo/Bajo & Alto/Alto & Bajo/Bajo & Medio/Alto \\
Norte & Alto/Alto & Alto/Alto & Bajo/Bajo & Medio/Bajo \\
Noreste & Bajo/Bajo & Bajo/Bajo & Alto/Alto & Bajo/Bajo \\
Centro-Norte & Bajo/Bajo & Medio/Medio & Bajo/Bajo & Medio/Medio \\
Centro-Occidente & Alto/Alto & Alto/Alto & Bajo/Bajo & Medio/Bajo \\
Centro-Este & Bajo/Medio & Bajo/Bajo & Medio/Bajo & Bajo/Bajo \\
Sur & Bajo/Alto & Alto/Alto & Alto/Alto & Alto/Medio \\
Este & Medio/Bajo & Alto/Alto & Medio/Medio & Bajo/Medio \\
Península de Yucatán & Bajo/Bajo & Bajo/Bajo & Alto/Alto & Alto/Alto \\
Sur-Sureste & Bajo/Medio & Alto/Alto & Alto/Bajo & Medio/Bajo \\
\hline & & & & \\
\hline Región & Índice & Índice & Índice & Índice de \\
& ambiental & apoyos públicos & competitividad & desarrollo \\
\hline Noroeste & Bajo/Bajo & Alto/Alto & Bajo/Bajo & Medio/Alto \\
Norte & Aedio/Medio & Alto/Alto & Alto/Alto & Medio/Medio \\
Noreste & Bajo/Bajo & Bajo/Alto & Alto/Alto & Alto/Alto \\
Centro-Norte & Bajo/Bajo & Bajo/Bajo & Bajo/Bajo & Medio/Medio \\
Centro-Occidente & Alto/Alto & Bajo/Bajo & Medio/Bajo & Medio/Medio \\
Centro-Este & Bajo/Bajo & Alto/Bajo & Alto/Medio & Medio/Medio \\
Sur & Alto/Alto & Bajo/Bajo & Alto/Alto & Bajo/Bajo \\
Este & Alto/Alto & Alto/Bajo & Medio/Bajo & Bajo/Medio \\
Península de Yucatán & Bajo/Bajo & Bajo/Alto & Bajo/Bajo & Medio/Medio \\
Sur-Sureste & Alto/Alto & Bajo/Bajo & Medio/Medio & Bajo/Bajo \\
\hline Fujo & Cojo & &
\end{tabular}

Fuente: las indicadas en el Cuadro 2.

Nota: los rangos de los índices de los factores y la competitividad correspondientes al periodo 1990-1995 son: Bajo: -0.31 a -0.18 ; Medio: -0.17 a -0.04 ; y Alto: -0.04 a 0.10. Los rangos correspondientes al periodo 1995-2000 son: Bajo: 0.70 a 0.76; Medio: .76 a 0.82; y Alto: 0.82 a 0.88 . 


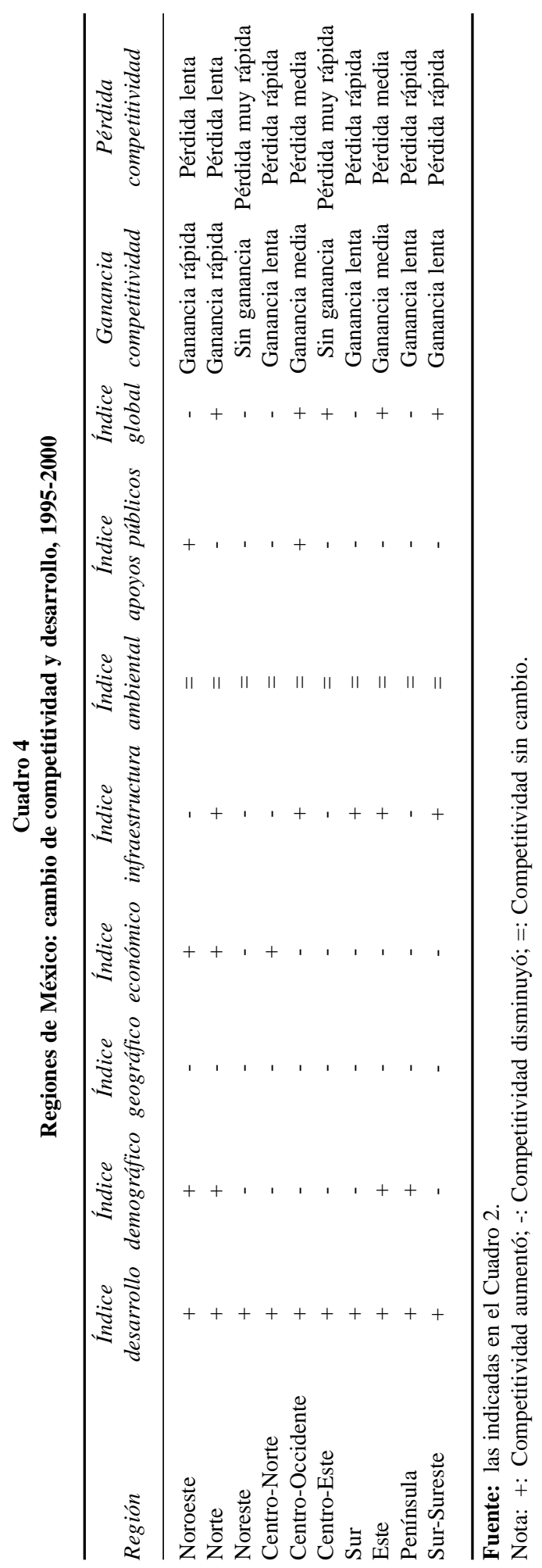

Desartar son 